\title{
Treatment of Angina and Microvascular Coronary Dysfunction
}

\author{
Arang Samim, MD, Lynn Nugent, MD, Puja K. Mehta, MD, Chrisandra Shufelt, MD, MS, and \\ C. Noel Bairey Merz, MD, FACC, FAHA \\ Cedars-Sinai Heart Institute, 444 South San Vicente Boulevard, Suite 600, Los Angeles, CA, \\ 90048, USA
}

\section{Opinion statement}

Microvascular coronary dysfunction (MCD) is an increasingly recognized cause of cardiac ischemia and angina, more commonly diagnosed in women. Patients with MCD present with the triad of persistent chest pain, ischemic changes on stress testing, and no obstructive coronary artery disease $(\mathrm{CAD})$ on cardiac catheterization. Data from National Heart, Lung and Blood Institute (NHLBI)-sponsored Women's Ischemia Syndrome Evaluation (WISE) study has shown that the diagnosis of MCD is not benign, with a $2.5 \%$ annual risk of adverse cardiac events including myocardial infarction, stroke, congestive heart failure, or death. The gold standard diagnostic test for MCD is an invasive coronary reactivity test (CRT), which uses acetylcholine, adenosine, and nitroglycerin to test the endothelial dependent and independent, microvascular and macrovascular coronary function. The CRT allows for diagnostic and treatment options as well as further risk stratifying patients for future cardiovascular events. Treatment of angina and MCD should be aimed at ischemia disease management to reduce risk of adverse cardiac events, ameliorating symptoms to improve quality of life, and to decrease the morbidity from unnecessary and repeated cardiac catheterization in patients with open coronary arteries. A comprehensive treatment approach aimed at risk factor managment, including lifestyle counseling regarding smoking cessation, nutrition and physical activity should be initiated. Current pharmacotherapy for MCD can include the treatment of microvascular endothelial dysfunction (statins, angiotensinconverting enzyme inhibitor, low dose aspirin), as well as treatment for angina and myocardial ischemia (beta blockers, calcium channel blockers, nitrates, ranolazine). Additional symptom management techniques can include tri-cyclic medication, enhanced external counterpulsation, autogenic training, and spinal cord stimulation. While our current therapies are effective in the treatment of angina and MCD, large randomized outcome trials are needed to optimize strategies to improve morbidity and mortality.

\section{Introduction}

Physicians often encounter patients with angina, abnormal stress testing, and no obstructive coronary artery disease (CAD), which is the triad for the suspicion for diagnosis of microvascular coronary dysfunction (MCD). Approximately $50 \%$ of women undergoing coronary angiography will have no obstructive CAD by angiography $[1,2]$. These patients have normal ventricular size and function and were previously diagnosed with Cardiac Syndrome X, which was believed to be a benign condition [3]. New data demonstrate that at least half of the patients diagnosed with Cardiac Syndrome X have MCD [4]. Data from the National Heart, Lung and Blood Institute (NHLBI) sponsored Women's Ischemia Syndrome

Corresponding author C. Noel Bairey Merz, MD, FACC, FAHA, Director, Women's Heart Center, Director, Preventive Cardiac Center, Heart Institute, Professor of Medicine, Cedars-Sinai Medical Center, 444 S San Vicente Blvd., Suite 600, Los Angeles, CA 90048, Mailing address, Noel.BaireyMerz@ cshs.org.

Disclosures

*Please see disclosure forms. 
Evaluation (WISE) study has demonstrated a $2.5 \%$ annual risk of adverse cardiac events including death, myocardial infarction, stroke or congestive heart failure [4,5] in subjects with MCD. Angina in the setting of MCD is believed to be due to myocardial ischemia. Invasive coronary reactivity testing (CRT), with the use of acetylcholine, adenosine, and nitroglycerin, is used to diagnose MCD, and to risk stratify patients for future cardiovascular events. In a complete CRT procedure, use of vasoactive substances, such as adenosine, acetylcholine, and nitroglycerin can detect abnormalities in both endothelial and nonendothelial pathways of coronary macro and microvascular dysfunction (Table 1). CRT can also be useful for differentiation of angina and MCD from Prinzmetal's angina and abnormal cardiac nociception [6]. While invasive coronary reactivity is considered gold standard for the diagnosis of MCD, non-invasive testing including adenosine stress cardiac magnetic resonance imaging (CMRI) to detect subendocardial ischemia, positron emission testing (PET) to detect abnormal coronary flow reserve, and single photon emission computed tomography (SPECT) can provide objective evidence suggestive of MCD.

Clinicians should consider MCD in the differential diagnosis in patients with persistent chest pain, objective evidence of myocardial ischemia either by stress testing or myocardial infarction but no obstructive CAD [7]. Failure to recognize MCD can lead to increased healthcare costs and increased patient morbidity from repeated non-invasive and invasive tests, as well as psychological stress from diagnostic and therapeutic uncertainty [8]. Once the diagnosis of MCD is considered, available therapies include medications to treat atherosclerosis and endothelial dysfunction (statin, angiotensin-converting enzyme inhibitor, low dose aspirin) and to reduce ischemia (beta blockers, calcium channel blockers, nitrates, ranolazine and enhanced external counterpulsation). This review focuses on current strategies for treatment of angina and MCD.

\section{Treatment}

Many of the known risk factors associated with MCD are similar to the known risk factors for obstructive $\mathrm{CAD}$, and include smoking, hypertension, diabetes, and hyperlipidemia. Aggressive treatment of risk factors appears indicated in the setting of MCD. There are a variety of pharmacological and non-pharmacological strategies for treatment of MCD (Figure 1), and many of the strategies have established evidence of benefit in CAD. Therapeutic lifestyle change regarding smoking cessation, nutrition and physical activity is important to improve endothelial dysfunction and anginal symptoms. Patient referral for exercise training in cardiac rehabilitation programs should also be strongly considered as it improves resting diastolic blood pressure, body mass index and exercise capacity in MCD patients [9].

Treatment for atherosclerosis and endothelial dysfunction (statin, angiotensin-converting enzyme inhibitor, low dose aspirin) is important. Statins improve endothelial function and decrease levels of C-reactive protein [10]. Angiotensin-converting enzyme inhibitors (ACEI) improve coronary flow reserve in patients with microvascular angina, likely mediated through their benefit on endothelial dysfunction [11, 12]. Angiotensin receptor blockers (ARBs) can be used in patients who are intolerant of ACE-Is, although no data is available in MCD subjects. Insulin resistance is has also been linked to endothelial dysfunction; use of metformin can improve microvascular function [13]. Pharmacologic therapy for MCD also includes the conventional anti-anginal medications of beta blockers, calcium channel blockers (CCB), nitrates and the newer anti-anginal medication ranolazine. A newer generation of beta-blockers with alpha-blocking properties may offer additional benefit in patients with angina and MCD. Although there is evidence that estrogen therapy improves endothelial function short-term in MCD subjects [14], hormone therapy increases the risk of 
adverse cardiac events in women with ischemic heart disease [15]. Estrogen therapy is not recommended for the treatment of cardiovascular disease including angina and MCD.

Non-pharmacological treatments can be used to improve MCD symptoms in patients who seek additional alternatives treatment options. Enhanced external counterpulsation (EECP) is an FDA-approved strategy for angina with obstructive CAD and therefore may be of benefit in angina and MCD [16]. Additional symptom management techniques include: regular exercise through cardiac rehabilitation, hypnosis in cases of high autonomic tone, and spinal cord stimulation in rare cases due to abnormal nociception. Regardless of the modality, therapy should be tailored to the individual patient based on their suspected underling pathophysiology, preference for pharmacotherapy, symptomatology, and motivation to participate in non-pharmacologic programs.

Below is a list of strategies for treatment of angina and MCD.

\section{Therapeutic Lifestyle Change [17]}

- Reduced intakes of saturated fats and cholesterol

- Therapeutic dietary options for enhancing LDL lowering (plant stanols/sterols and increased viscous [soluble] fiber

- A Mediterranean style diet, with olive oil as the principal source of fat, use of fish over red meat, and abundance of fresh unprocessed fruits and vegetables, has been shown to improve endothelial function and inhibit oxidative stress [18].

- Weight reduction

- Regular moderate intensity physical activity for 30 minutes on most, if not all, days of the week

- Smoking cessation

- Moderate alcohol intake (no more than one drink per day in women, no more than two drinks per day in men)

\section{Pharmacologic Treatment}

\section{Aspirin}

A high percentage of patients with MCD have atherosclerosis detected by coronary intravascular ultrasound (IVUS); therefore, aspirin should be considered for secondary prevention of cardiovascular events in patients with MCD [19].

\section{Beta-Blockers}

Beta-blockers are effective in reducing frequency and severity of anginal symptoms [20] and can be used effectively in treatment of angina in MCD. Beta blockers that also have alpha blocking activity (labetolol, carvediolol, nebivolol) may be preferred in patients with MCD since these agents have been shown to improve CFR in the setting of ischemic heart disease [21]. Both carvediolol and nebivolol have anti-oxidant properties that improve endothelial function [22, 23]; however, there is a lack of randomized controlled trial data in patients with MCD comparing these individual agents at this time.

\section{Calcium Channel Blockers}

CCBs selectively block the transmembrane influx of calcium ions into arterial smooth muscles. Their antihypertensive effect is through the reduction of systemic vascular resistance and vasodilation of peripheral arteries. CCBs are recommended as first line 
therapy for Prinzmetal's angina [20] because they inhibit coronary vasospasm. Diltiazem has not been shown to improve CFR in MCD [26], and in direct comparison trials, beta blockers were found to be more effective than CCBs in symptom control of angina [24, 25]. Among patients with Prinzmetal's angina, symptoms may worsen with beta-blockers and require CCBs for symptom control.

\section{Nitrates}

Along with beta-blockers and CCBs, nitrates can be used in angina management. They stimulate cGMP production which relaxes vascular smooth muscle cells, cause dilatation of peripheral arteries and veins, and relieve angina. Nitrates are available in a variety of formulations, from short acting sublingual tablets to long acting topical patches. Sublingual nitrates may be effective in approximately $50 \%$ of patients with angina and MCD [27]. Nitrate free intervals are important since a major limitation of their use is the development of tolerance in many patients.

\section{Other Anti-Anginals: Ranolazine}

Ranolazine is a novel anti-anginal therapy that is FDA-approved for treatment of chronic stable angina in patients with CAD [28]. Although the various mechanistic actions are unclear, it is thought to inhibit the late sodium current of the cardiac action potential. In ischemic myocardium, intracellular acidosis impairs sodium homeostasis and leads to calcium overload. Inhibition of sodium entry leads to improved sodium and calcium homeostasis and improved anginal symptoms [29]. Ranolazine is unique in that it produces anti-anginal effect without hemodynamic consequences of altering blood pressure or heart rate. This medication can be considered in patients with refractory angina and used as supplemental therapy to a regimen of beta blockers, CCBs, and nitrates, or as first line therapy in patients with contra-indications to traditional anti-anginal medications.

\section{HMG-COA Reductase Inhibitors (Statins)}

Statins inhibit the conversion of HMG-CoA to mevalonate, which is the rate limiting step in the biosynthesis of cholesterol. Reduced cholesterol synthesis also causes an increase in LDL receptor synthesis, which further lowers serum LDL. Beyond the LDL lowering effects, statins have been shown to improve CFR [30], and improve exercise tolerance and reduce angina, likely due to anti inflammatory effects on endothelial function [10]. Patients with MCD should be considered for treatment to a goal LDL of less than 100, and ideally 70 , given the high prevalence of coronary atherosclerosis by IVUS, as per the current guidelines for secondary prevention [17].

\section{ACE inhibitors/Angiotensin Receptor Blockers}

ACE-Is inhibit the conversion of angiotensin I to angiotensin II, the potent, primary effector of the renin-angiotensin-aldosterone system, which is involved in several cardiovascular processes, including blood pressure regulation, endothelial function, and vascular inflammation. ACE-Is can be used in MCD to improve coronary endothelial function [11], and have been shown to improve exercise duration and CFR in patients with MCD [31]. A majority of patients with MCD are women, and pregnancy counseling is critical when prescribing ACE-I to women, due to their known teratogenic effects. ARBs have also been shown to improve CFR [32], and should be used in patients who are intolerant to ACE-Is, but they are relatively more expensive as most are still on patent, however losartan has recently become generic. 


\section{Tri-Cyclic Medications}

Tricyclics such as imipramine and amitriptyline block multiple receptors, including muscarinic acetylcholine receptors, $\mathrm{H} 1$ and $\mathrm{H} 2$ histamine receptors, sodium and calcium channel receptors. They also inhibit reuptake of serotonin and norepinephrine. They are used to treat patients with chest pain due to abnormal cardiac nociception. In a randomized, placebo-controlled trial of patients with angina but no obstructive coronary artery disease, imipramine has been shown to reduce frequency of anginal episodes [33].

\section{Physical Activity and Therapy}

- Exercise has clearly been shown to improve endothelial function and improve exercise capacity [9]

- Patients who are part of a well documented exercise program such as cardiac rehabilitation have improved quality of life, psychological morbidity and symptom severity [9].

\section{Cardiac Rehabilitation Program}

Cardiac rehabilitation programs involve a multidisciplinary approach with cardiovascular exercise, nutritional education, and psychological counseling. The program usually includes 36 telemetry monitored sessions completed over a twelve week period. Patient compliance and motivation to complete such a program is the key to their success. Patients who complete the 36-monitored session phase of cardiac rehabilitation may progress in a maintenance program to continue their progress. The costs of these programs vary depending on insurance coverage and the program. A physician prescription is needed for referral to cardiac rehabilitation and angina is a covered diagnosis for most insurers.

\section{Autogenic Training}

Autogenic training is a hypnosis-based relaxation technique consisting of six standard exercises aimed at reducing autonomic anger and stress symptoms [34]. The goal is for the patient to gain more control over autonomic functions, improve anxiety and reduce heart rate and blood pressure, resulting in improved symptoms and quality of life.

\section{Other Treatments}

- Enhanced external counterpulsation (ECP) is an approved non-invasive therapy for angina which improves myocardial ischemia and endothelial function, and can be used in patients with angina due to $\mathrm{MCD}[16]$.

- Invasive, non-pharmacological interventions include transcutaneous electrical nerve stimulation (TENS) and spinal cord stimulation are effective albeit last resort pain management strategies. These therapie are aimed at treating the abnormal excitability of the nociceptive system [35]. Spinal nerve stimulation may be able to restore habituation to peripheral nerve stimuli [36]

\section{Enhanced External Counterpulsation}

ECP can be used for treatment of angina that is unresponsive to standard therapies. Pneumatic stockings are placed on the patient's lower extremities and inflated and deflated during diastole and systole, respectively. The aim is to reduce afterload and to increase preload to improve endothelial function and promote collateral coronary flow. Sessions last for one hour, five times per week and is continued for up to 2 months. It is contraindicated in patients with severe peripheral vascular disease and deep vein thrombosis. The procedure is generally well tolerated, but side effects include persistent muscle aches, fatigue, and 
dizziness. It is a time consuming method that requires many sessions, but in adherent patients with refractory angina it has been shown to improve symptoms [16]. The cost varies as well, depending on the program and insurance coverage, but angina is a covered diagnosis for many insurers.

\section{Spinal Cord Stimulation}

Implantation of spinal cord stimulator device has been used in select patients with refractory cardiac chest pain who are thought to have abnormal nociception [36]. It cannot be used in patients who have implanted cardiac devices or who are on anticoagulation therapy. Complications related to the procedure include epidural abscess formation, bleeding, and neurological damage. It is significantly more expensive than conventional therapies, but can be considered to control symptoms and reduce hospitalizations and repeated, unnecessary cardiac testing.

\section{Emerging Therapies}

- PDE-5 inhibitors, such as sildenaphil, may be appropriate in some patients who have persistent MCD unresponsive to traditional anti-anginals and can avoid longacting nitrates [37].

- L-arginine, a precursor of nitric oxide, is emerging as a potential therapeutic agent due to its beneficial effect on endothelial function by promoting release of nitric oxide, and by reducing levels of endothelin-1, a potent vasoconstrictor [38]. However, it increases mortality in post myocardial infarction patients [39] and its safety in MCD patients is unknown.

\section{Conclusions}

Women with angina, objective evidence of ischemia, and no obstructive CAD have a high prevalence of MCD. Angina and MCD is associated with adverse cardiac morbidity and mortality, and treatment should be aimed at ameliorating symptoms to improve quality of life, decrease the morbidity from unnecessary and repeated cardiac catheterization, and reduce risk of adverse cardiac events. Treatment of angina and MCD can include therapeutic lifestyle change including smoking cessation, nutrition and physical activity; medications to treat atherosclerosis and endothelial dysfunction (statin, ACE-I, low dose aspirin), as well as medications to reduce angina and ischemia (beta blockers, CCB, nitrates, ranolazine). Additional symptom management techniques can include tri-cyclic medication, autogenic training, ECP, spinal cord stimulation and possibly PDE-5 inhibitors and L-arginine. While our current therapies are effective in the treatment of angina and MCD, large randomized outcome trials are needed to optimize strategies for morbidity and mortality.

\section{Acknowledgments}

This work was supported by contracts from the National Heart, Lung and Blood Institutes, nos. N01-HV-68161, N01-HV-68162, N01-HV-68163, N01-HV-68164, a GCRC grant MO1-RR00425 from the National Center for Research Resources, and grants from the Gustavus and Louis Pfeiffer Research Foundation, Denville, New Jersey, The Women's Guild of Cedars-Sinai Medical Center, Los Angeles, California, and The Ladies Hospital Aid Society of Western Pennsylvania, Pittsburgh, Pennsylvania, the Edythe L. Broad Women's Heart Research Fellowship, Cedars-Sinai Medical Center, Los Angeles, California, and the Barbra Streisand Women's Cardiovascular Research and Education Program, Cedars-Sinai Medical Center, Los Angeles.

\section{References and Recommended Reading}

Papers of particular interest, published recently, have been highlighted as: 
- Of importance

-• Of major importance

1••. Sharaf BL, Pepine CJ, Kerensky RA, et al. Detailed angiographic analysis of women with suspected ischemic chest pain(pilot phase data from the NHLBI-sponsored Women's Ischemia Syndrome Evaluation [WISE] Study Angiographic Core Laboratory). Am J Cardiol. 2001; 87:937-41. [PubMed: 11305981]

2. Likoff W, Segal BL, Kasparian H. Paradox of normal selective coronary arteriograms in patients considered to have unmistakable coronary heart disease. N Engl J Med. 1967; 267:1063-6. [PubMed: 6025663]

3. Pasternak RC, Thibault GE, DeSanctis RW, Hutter AM. Chest pain with angiographically insignificant coronary arterial obstruction: clinical presentation and long-term follow up. Am J Med. 1980; 68:813-7. [PubMed: 7386488]

4. Reis SE, Holubkov R, Conrad Smith AJ, Kelsey SF, Sharaf BL, Reichek N, Rogers WJ, Merz CN, Sopko G, Pepine CJ. WISE Investigators. Coronary microvascular dysfunction is highly prevalent in women with chest pain in the absence of coronary artery disease: results from the NHLBI WISE study. Am Heart J. 2001; B141(5):735-41. [PubMed: 11320360]

5. Johnson BD, Shaw LJ, Buchthal SD, et al. Prognosis in women with myocardial ischemia in the absence of obstructive coronary disease, Results from the National Institutes of Health-National Heart, Lung, and Blood Institute-sponsored Women's Ischemia Syndrome Evaulation(WISE). Circulation. 2004; 109:2993-9. [PubMed: 15197152]

6••. Johnson BD, Shaw LJ, Pepine CJ, et al. Persistent chest pain predicts cardiovascular events in women without obstructive coronary artery disease: Results from the NIH-NHLBI-sponsored Women's Ischaemia Syndrome Evaluation (WISE) study. Eur Heart J. 2006; 27:1408-1415. [PubMed: 16720691]

7•. Cannon R. Microvascular Angina and the continuing Dilemma of Chest Pain with Normal Coronary Angiograms. JACC. 2009; 54:877-87. A thorough review of the differing literature on pathophysiology and treatment of microvascular angina. [PubMed: 19712795]

8. Shaw LJ, Merz CN, Pepine CJ, Reis SE, Bittner V, Kip KE, Kelsey SF, Olson M, Johnson BD, Mankad S, Sharaf BL, Rogers WJ, Pohost GM, Sopko G. Women's Ischemia Syndrome Evaluation (WISE) Investigators. The economic burden of angina in women with suspected ischemic heart disease: results from the National Institutes of Health--National Heart, Lung, and Blood Institute-sponsored Women's Ischemia Syndrome Evaluation. Circulation. 2006; 114(9):894-904. [PubMed: 16923752]

9. Asbury EA, Slattery C, Grant A, et al. Cardiac rehabilitation for the treatment of women with chest pain and normal coronary arteries. Menopause. 2008; 15(3):454-460. [PubMed: 18188136]

10. Kayikcioglu M, Payzin S, Yavuzgil O, et al. Benefits of statin treatment in cardiac syndrome-X1. Eur Heart J. 2003; 24:1999-2005. [PubMed: 14613735]

11. Kaski JC, Rosano G, Gavrielides S, et al. Effects of angiotensin-converting enzyme inhibition on exercise-induced angina and ST segment depression in patients with microvascular angina. J Am Coll Cardiol. 1994; 23:652-657. [PubMed: 8113548]

12. Pizzi C, et al. Angiotensin-converting enzyme inhibitors and 3-hydroxy-3-methylglutaryl coenzyme A reductase in cardiac Syndrome X: role of superoxide dismutase activity. Circulation. 2004; 109(1):53-8. [PubMed: 14699004]

13. Jadhav $\mathrm{S}$, et al. Effects of metformin on microvascular function and exercise tolerance in women with angina and normal coronary arteries: a randomized, double-blind, placebo-controlled study. J Am Coll Cardiol. 2006; 48(5):956-63. [PubMed: 16949486]

14. Roque M, Heras M, Raig E, et al. Short-term effects of transdermal oestrogen replacement therapy on coronary vascular reactivity in postmenopausal women with angina pectoris and normal results on coronary angiograms. J Am Coll Cardiol. 1998; 31:139-43. [PubMed: 9426032]

15. Hsia, Judith, MD; Langer, Robert D., MD, MPH; Manson, JoAnn E., MD, DrPH; Kuller, Lewis, MD, DrPH; Johnson, Karen C., MD, MPH; Hendrix, Susan L., DO; Pettinger, Mary, MS; Heckbert, Susan R., MD, PhD; Greep, Nancy, MD; Crawford, Sybil, PhD; Eaton, Charles B., MD; Kostis, John B., MD; Caralis, Pat, MD; Prentice, Ross, PhD. for the Women's Health Initiative 
Investigators. Conjugated Equine Estrogens and Coronary Heart Disease. Arch Intern Med. 2006; 166:357-365. [PubMed: 16476878]

16. Kronhaus KD, Lawson WE. Enhanced External Counterpulsation is an effective treatment for syndrome X. Int J Cardiol. 2009; 135(2):256-7. [PubMed: 18590931]

17. Third Report of the NCEP Expert Panel on Detection, Evaluation, Treatment, of High Blood Cholesterol in Adults (Adult Treatment Panel III) Final Report. Circulation. 2002 Dec 17; 106(25):3143-421. [PubMed: 12485966]

18. Parikh P, McDaniel MC, Ashen D, et al. Diets and Cardiovascular Disease; An Evidence Based Assessment. J Am Coll Cardiol. 2005; 45:1379-87. [PubMed: 15862406]

19. Baigent C, Blackwell L, Collins R, et al. Aspirin in the primary and secondary prevention of vascular disease: collaborative meta-analysis of individual participant data from randomised trials. Lancet. 2009; 373:1849-1860. [PubMed: 19482214]

20. Fraker TD Jr, Fihn SD, Gibbons RJ, et al. 2007 chronic angina focused update of the ACC/AHA 2002 Guidelines for the management of patients with chronic stable angina: a report of the American College of Cardiology/American Heart Association Task Force on Practice Guidelines Writing Group to develop the focused update of the 2002 Guidelines for the management of patients with chronic stable angina. Circulation. Dec 4; 2007 116(23):2762-2772. [PubMed: 17998462]

21. Kaski JC, Rodriguez-Plaza L, Brown J, et al. Efficacy of carvedilol (BM14, 190), a new betablocking drug with vasodilating properties, in exercise-induced ischemia. Am J Cardiol. $1985 \mathrm{Jul}$ 1; 56(1):35-40. [PubMed: 2861738]

22. Matsuda Y, Akita H, Terashima M, et al. Carvedilol improves endothelium-dependent dilation in patients with coronary artery disease. Am Heart J. 2000 Nov; 140(5):753-9. [PubMed: 11054621]

23. Sen N, Tavil Y, Erdamar H, et al. Nebivolol therapy improves endothelial function and increases exercise tolerance in patients with cardiac syndrome X. Anadolu Kardiyoloji Dergisi. 2009; 9(5): 371-9. [PubMed: 19819787]

24. Lanza GA, Colonna G, Pasceri V, et al. Atenolol versus amlodipine versus isosorbide-5mononitrate on anginal symptoms in syndrome X. Am J Cardiol. 1999; 84:854-6. [PubMed: 10513787]

25. Bugiardini R, et al. Comparison of verapamil versus propranolol therapy in syndrome X. Am J Cardiol. 1989; 63(5):286-90. [PubMed: 2643845]

26. Bairey Merz CN, Eteiba W, Pepine CJ, et al. Cardiac Syndrome X: Relation to Microvascular Angina and Other Conditions. Curr CV Risk Reports. 2007; 1:167-175.

27. Kaski JC, Rosano GM, Collins P, et al. Cardiac Syndrome X: clinical characteristics and Left ventricular function. Long-term follow-up study. J Am Coll Cardiol. 1995; 25:807-814. [PubMed: 7884081]

28. Chapman BR. Ranolazine for the Treatment of Chronic Angina and Potential Use in Other Cardiovascular Conditions. Circulation. 2006; 113:2462-2472. [PubMed: 16717165]

29. Chaitman BR. Ranolazine for the treatment of chronic angina and potential use in other cardiovascular conditions. Circulation. May 23; 2006 113(20):2462-2472. [PubMed: 16717165]

30. Caliskan M, Erdogan D, Gullu H, et al. Effects of atorvastatin on coronary flow reserve in patients with slow coronary flow. Clin Cardiology. Sep; 2007 30(9):475-479.

31. Chen JW, Hsu NW, Wu TC, Lin SJ, Chang MS. Long-term angiotensin-converting enzyme inhibition reduces plasma asymmetric dimethylarginine and improves endothelial nitric oxide bioavailability and coronary microvascular function in patients with syndrome X. Am J Cardiol. Nov 1; 2002 90(9):974-982. [PubMed: 12398965]

32. Higuchi T, Abletshauser C, Nekolla SG, et al. Effect of the angiotensin receptor blocker Valsartan on coronary microvascular flow reserve in moderately hypertensive patients with stable coronary artery disease. Microcirculation. 2007; 8:805-812. [PubMed: 17907015]

33. Cannon RO III, Quyyumi AA, Mincemoyer R, et al. Imipramine in patients with chest pain despite normal coronary angiograms. N Engl J Med. 1994; 330:1411-1417. [PubMed: 8159194]

34. Asbury EA, Kanji N, Ernst E, et al. Autogenic training to manage symptomology in women with chest pain and normal coronary arteries. Menopause. 2009; 16(1):60-65. [PubMed: 18978640] 
35. Valeriani M, Sestito A, Le Pera D, et al. Abnormal cortical pain processing in patients with cardiac syndrome X. Eur Heart J. 2005; 26:975-82. [PubMed: 15790583]

36. Sestito A, Lanza GA, Le Pera D, et al. Spinal Cord Stimulation normalizes abnormal cortical pain processing in patients with cardiac syndrome X. Pain. 2008; 139:82-89. [PubMed: 18440702]

37. Radovits T, Bomicke T, Kokeny G, et al. The phosphodiesterase-5 inhibitor vardenafil improves cardiovascular dysfunction in experimental diabetes mellitus. Br J Pharmacol. 2009; 156(6):909919. [PubMed: 19298393]

38. Piatti P, Fragasso G, Monti LD, et al. Acute intravenous L-arginine infusion decreases endothelin-1 levels and improves endothelial function in patients with angina pectoris and normal coronary arteriograms: correlation with asymmetric dimethylarginine levels. Circulation. 2003; 107(3):429-36. [PubMed: 12551867]

39. Schulman SP, Becker LC, Kass DA, et al. L-Arginine Therapy in Acute Myocardial Infarction. JAMA. 2006; 295:58-64. [PubMed: 16391217] 


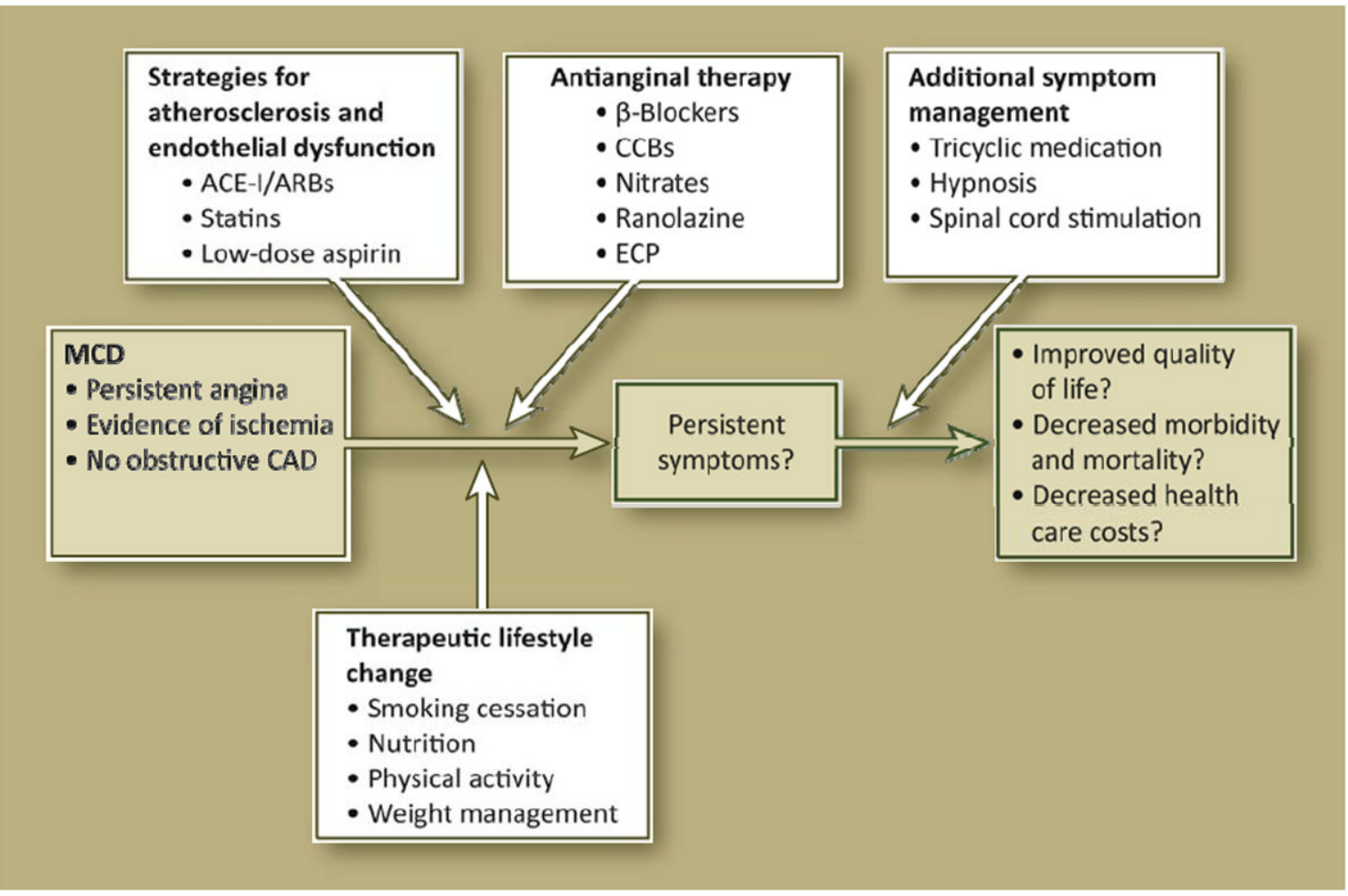

Figure 1.

Algorithm for the treatment of angina and microvascular coronary dysfunction. ACE-I angiotensin-converting enzyme inhibitors; ARBs angiotensin receptor blockers; CAD coronary artery disease; CCBs calcium channel blockers; ECP external counterpulsation; MCD microvascular coronary dysfunction. 
